\title{
Investigation of a measles outbreak in Zimbabwe, 2010: potential of a point of care test to replace laboratory confirmation of suspected cases
}

\author{
A. SHONHAI ${ }^{1}$, L. WARRENER $^{2}$, D. MANGWANYA ${ }^{3}$, R. SLIBINSKAS ${ }^{4}$, \\ K. BROWN ${ }^{2}$, D. BROWN ${ }^{2}$, D. FEATHERSTONE ${ }^{5}$ AND D. SAMUEL ${ }^{2 *}$ \\ ${ }^{1}$ Zimbabwe National Virology Laboratory, Department of Medical Microbiology, College of Health Science, \\ University of Zimbabwe, Zimbabwe \\ ${ }^{2}$ Virus Reference Department, Public Health England, London, UK \\ ${ }^{3}$ Ministry of Health and Child Care, Zimbabwe \\ ${ }^{4}$ Institute of Biotechnology of Vilnius University, Vilnius, Lithuania \\ ${ }^{5}$ World Health Organization: Expanded Programme on Immunization (EPI), Department of Immunization, \\ Vaccines and Biologicals (IVB), Geneva, Switzerland
}

Received 11 December 2014; Final revision 9 February 2015; Accepted 27 February 2015; first published online 13 April 2015

\section{SUMMARY}

Blood and oral fluid (OF) samples were collected from 103 suspected measles cases between February and November 2010 during a nationwide measles outbreak in Zimbabwe. Siemens measles IgM enzyme immunoassay (EIA) on serum, Microimmune measles IgM capture EIA on OF, real-time haemagglutinin $(\mathrm{H})$ gene PCR and nested nucleocapsid $(\mathrm{N})$ gene PCR on OF were performed, confirming 75 measles cases. These samples were then used to evaluate a newly developed point of care test (POCT) for measles and determine its potential for identifying measles cases in outbreaks. After performing POCTs on OF samples, nucleic acid was extracted from the used test strips and the measles $\mathrm{H}$ and $\mathrm{N}$ genes amplified by RT-PCR. The sensitivity, specificity, positive and negative predictive values of the POCT for IgM in OF was $75 \cdot 0 \%[95 \%$ confidence interval (CI) 63.4-84·5], 96.2\% (95\% CI 80.4-99.9), 98.2\% (95\% CI 90-3-100) and $58 \cdot 1 \%(95 \%$ CI $42 \cdot 1-73 \cdot 0)$, respectively. The $\mathrm{N}$ gene sequences showed high level of agreement between original OF and corresponding POCT strips. Measles genotype B3 was identified in all cases. We conclude that the measles POCT has the potential to be used, at the point of contact, in outbreak situations and provide molecular characterization of the virus at a later date.

Key words: Genotype, IgM, measles, oral fluid, point of contact.

\section{INTRODUCTION}

Measles is vaccine preventable and yet figures from the World Health Organization (WHO) estimated that in 2010 it contributed to over 139000 deaths globally, mostly in young children [1]. Declining

\footnotetext{
* Author for correspondence: Dr D. Samuel, Virus Reference Department, Public Health England, 61 Colindale Avenue, London NW9 5EQ, UK.

(Email: dhan.samuel@phe.gov.uk)
}

immunization coverage in Zimbabwe between 1996 and 2004 combined with measles outbreaks in Eastern and Southern Africa, including neighbouring South Africa, in 2009, led to outbreaks of measles in Zimbabwe from September 2009 to 2010. A nationwide measles immunization campaign took place between 24 May and 2 June 2010. Surveillance of clinical cases and laboratory confirmation of measles was an important tool in mobilizing the immunization campaign. 
Laboratory confirmation of measles is by detection of measles-specific IgM in serum samples [2,3] or in dried blood spots [4, 5] and oral fluid (OF) [6]. Detection of viral ribonucleic acid (RNA) in OF specimens collected soon after clinical presentation has also been employed for the early diagnosis of measles, and for molecular characterization of the strain of measles virus causing the disease to track transmission pathways and identify importations [7, 8].

Recently, we described the development of a point of care test (POCT) for measles to speed identification, enhance surveillance and reduce response time in resource-poor settings [9]. In this paper we describe the performance of the POCT on serum and OF samples obtained from suspected cases of measles occurring in Zimbabwe, between February and November 2010 , to assess its potential to confirm outbreaks.

\section{METHODS}

\section{Specimen collection (Zimbabwe)}

Matched blood samples and OF specimens were collected from 96/103 suspected measles cases between February and November 2010 during a nationwide outbreak in Zimbabwe. For seven suspected cases only OF specimens were collected. Blood specimens were collected by venepuncture and OF using the Oracol $^{\mathrm{TM}}$ collection device (Malvern Medical Development, UK). The geographical distribution of cases by province was Harare $(n=38)$, Chitungwiza $(n=23)$, Mashonaland East $(n=19)$, Mashonaland Central $(n=6)$, Masvingo $(n=6)$, Midlands $(n=6)$, Mashonaland West $(n=4)$ and Manicaland $(n=1)$.

The Expanded Programme on Immunization (EPI) measles surveillance network was used to collect samples from suspected cases as they reported to health centres or when district nurses collected samples during visits to areas where there were suspected cases. The Oracol $^{\mathrm{TM}}$ swabs were distributed to districts reporting outbreaks. Health centre staff and district nurses were informed of the study and trained to collect OF samples. OF samples were transported within $24 \mathrm{~h}$ of collection to the Zimbabwe National Virology Laboratory in Harare, together with a matched blood sample, using the EPI cold chain system. Serum was separated from whole blood samples within $24 \mathrm{~h}$ of collection using standard methods [10] and stored at $-20^{\circ} \mathrm{C}$. The sera were tested by measles IgM enzyme immunoassay (EIA) within 7 days of collection. The OF swabs received in the laboratory were frozen at $-80^{\circ} \mathrm{C}$ until processed. OF swabs, received before July 2010, were processed in July 2010 and swabs received after July 2010 were processed in April 2011 using the WHO's standard protocol [10]. After extracting the OF from the swabs, the OF specimens were stored between 2 and $8^{\circ} \mathrm{C}$ for up to 7 days and used for measles IgM EIA testing. Serum and OF samples were frozen at $-80{ }^{\circ} \mathrm{C}$ before being transported on dry-ice to the UK for further testing.

Blood and OF swabs from each subject were collected on the same day, except in five cases. For three subjects, the OF swabs were collected 1, 2 and 6 days prior to blood collection. In an additional two cases, blood and OF swab collection dates were not recorded. For seven subjects, only an OF swab was collected, giving 96 OF swabs with matched blood samples.

Timing of specimens in relation to onset of symptoms was available for $88 / 103$ subjects, all of which had been collected within 7 days of onset of symptoms (61 within 0-3 days of onset). For the remaining 15 individuals either the date of onset of symptoms or the collection dates were not known or not recorded.

Ages were recorded for 93/103 cases and were between 3 months and 49 years, with a mean age of $14 \cdot 6$ years (geo mean 7.9 years). This included 17 individuals, aged 9 months to 14 years, who between August 2009 and September 2010 had received one dose of measles containing vaccine, ranging from 266 to 2 days prior to onset of rash. Three of these cases received vaccine during the catch-up campaign and 10 others received the vaccine in June 2010.

\section{EIA}

Serum and OF specimens were tested initially in Zimbabwe for measles-specific IgM using the Enzygnost anti-measles virus/IgM (Siemens Healthcare Diagnostic Products GmbH, Germany) and Measles IgM Capture EIA (Microimmune Ltd, UK), respectively. Eighty-seven of 96 sera were also tested using the Enzygnost anti-rubella virus/IgM EIA (Siemens Healthcare Diagnostic Products $\mathrm{GmbH}$ ) in the UK and results for these are also reported here.

\section{POCT}

The POCT for measles IgM detection was performed in the UK on 94 sera and 99 OF samples. After testing for measles IgM EIA, insufficient volume of specimen was available for testing by POCT for two cases from 
whom serum was collected and from four cases where OF was collected.

The POCT strip used for measles-specific IgM detection was formatted as described previously [9]. Briefly, POCT strips consist of three distinct areas, each having a different function in the assay. At one end of the POCT strip is a conjugate release pad (CRP) impregnated with $40 \mathrm{~nm}$ gold particles labelled with a monoclonal antibody to measles nucleoprotein, which is inserted into a tube containing a mixture of the sample and measles antigen. The CRP overlaps the middle, analytical section of the test strip consisting of a nitrocellulose membrane with anti-human IgM test line and anti-mouse $\operatorname{IgG}$ control line dispensed as discrete, immobile bands approximately $14 \mathrm{~mm}$ and $18 \mathrm{~mm}$, respectively, from the CRP. Finally an absorbent paper wick overlaps the nitrocellulose membrane at the distal end.

To perform the POCT, diluted serum or undiluted OF is first mixed with recombinant measles nucleoprotein (rMVN) antigen in a tube as described previously [9]. The POCT strip is then inserted into the test specimen, initiating sample flow up the strip by capillary action.

After $20 \mathrm{~min}$ incubation at room temperature results were recorded by three independent observers, who were all experienced laboratory workers. Results were interpreted as valid when the pink/red control line was visible, and positive for measles-specific $\operatorname{IgM}$ if at least two of three observers recorded a visible pink/red band at the anti-human IgM test line, or negative if at least two observers recorded the absence of a visible band at the test line.

\section{Real-time measles $\mathrm{H}$ gene PCR and nested $\mathbf{N}$ gene PCR}

OF samples from 102 suspected measles cases and 100 POCT strips, after being used for IgM detection in $\mathrm{OF}$, were available for nucleic acid extraction and amplification. Nucleic acid extraction [11], cDNA preparation, real-time measles $\mathrm{H}$ gene $\mathrm{PCR}$ and nested $\mathrm{N}$ gene PCR were performed in the UK, according to procedures described previously [12-15]. As a control for specimen adequacy amplification of the human Beta-2 microglobulin (B2M) gene was performed simultaneously with real-time PCR for measles $\mathrm{H}$ gene on all OF specimens and also for specimens tested by POCT and extracted from the POCT strips. The real-time PCR preferentially amplified measles $\mathrm{H}$ gene so that low levels of measles viral nucleic acids were not out-competed by $B 2 M$. Real-time PCR results on OF were interpreted as negative if the $\mathrm{H}$ gene was not amplified but the $B 2 M$ gene was detected. The results were interpreted as not detected, if both $\mathrm{H}$ gene and $B 2 M$ genes were not amplified.

\section{Laboratory-confirmed case}

For the purpose of this investigation, amplification of measles $\mathrm{H}$ and/or $\mathrm{N}$ gene by PCR directly on OF, or a positive measles IgM EIA result on serum from a suspected case was taken as confirming measles.

\section{Genotyping of measles strains}

Amplified $\mathrm{N}$ gene products obtained after amplification from 29 OF specimens and from 25 POCT strips used for IgM detection in $\mathrm{OF}$ were sequenced by dideoxynucleotide sequencing in a Genetic Analyser (Applied Biosystems, USA), analysed with Bionumerics software v. 6.1 (Applied Maths NV, Belgium) and genotyped by comparison with reference strain sequences according to WHO guidelines [16].

\section{RESULTS}

\section{Measles IgM detection by POCT on OF from suspected cases}

OF samples were collected from all 103 suspected measles cases and serum from 96 of these subjects. After testing OF using the Microimmune measles IgM capture EIA, sufficient OF specimens were available for testing with POCT from 99/103 suspected cases. For 92/99 cases, serum samples were also available for testing with the Siemens measles IgM EIA.

The results for these 92 cases are shown in Table 1 . There was agreement between the three readers in interpreting the results of POCT on all OF samples and the majority of serum samples. For four serum samples two of three readers agreed on the POCT results and these were interpreted as described in the Methods section. The POCT detected measles IgM in $47 / 57$ cases that were positive in the serum EIA and 29/31 cases that were serum IgM negative were also negative by POCT. OF from $2 / 4$ cases giving serum EIA equivocal results were positive for IgM by POCT. The sensitivity and specificity of the POCT on OF for measles IgM detection compared to the EIA on serum were $83.9 \%$ and $93.5 \%$, respectively. 
Table 1. Comparison of the detection of measles specific IgM in serum by EIA and in OF by POCT

\begin{tabular}{lrrr}
\hline \hline & \multicolumn{3}{l}{$\begin{array}{l}\text { Measles IgM detection in OF } \\
\text { using POCT }\end{array}$} \\
\cline { 2 - 4 } Serum IgM EIA results & Positive & Negative & Total \\
\hline Positive & 47 & 10 & 57 \\
Equivocal & 2 & 2 & 4 \\
Negative & 2 & 29 & 31 \\
Total & 51 & 41 & 92 \\
\hline \hline
\end{tabular}

EIA, Enzyme immunoassay; OF, oral fluid; POCT, point of care test.

Serum specimens were tested using the Siemens Enzygnost anti-measles virus/IgM EIA.

OF samples from the 47 cases that were positive in both the POCT and serum EIA were also positive in Microimmune measles IgM EIA, confirming measles in these cases. Measles $\mathrm{H}$ and $\mathrm{N}$ genes were detected by PCR in OF in 45/47 cases. The first of the two measles IgM-positive cases which was negative by PCR was from a 13-year-old whose date of onset was not known. The second case was a 5-month-old whose specimens were collected 2 days post-onset of rash symptoms, and their OF was very strongly positive in the measles IgM EIA $\left(\mathrm{OD}_{450 \mathrm{~nm}} 2 \cdot 265\right.$, test/cutoff $>25 \cdot 0$ ). Further, the measles $\mathrm{N}$ gene was amplified and genotyped as B3 from POCT strips that had been used for IgM testing of the OF but it was not amplified directly from the OF.

OF from 8/10 serum IgM-positive, POCT-negative cases were positive in the Microimmune measles IgM capture EIA and one of the two remaining cases gave an equivocal result. $\mathrm{H}$ and $\mathrm{N}$ genes were amplified by PCR in all 10 of these cases. In addition, $\mathrm{H}$ and $\mathrm{N}$ genes were amplified from $\mathrm{OF}$ in the four serum IgM EIA equivocal cases, shown in Table 1, and from a further serum IgM EIA equivocal case for whom insufficient volume of OF was available for testing by POCT.

Two of the 31 serum IgM EIA-negative cases gave a positive result in the OF POCT. The OF from each case was PCR negative. One of these cases was a 17-month-old child who had received measles vaccine 266 days prior to sample collection, which indicates a false positive result in the POCT. The other case was an 8-year-old from whom the OF sample was taken 7 days post-onset of rash; the measles IgM EIA result on this sample was also positive. Although this case may be a true positive, with viral nucleic acid declining to undetectable levels at day 7 , it was interpreted as a false positive according to the criteria used here for laboratory-confirmed measles case.

Of the 29 cases that were measles IgM negative by both serum EIA and POCT on OF, $\mathrm{H}$ and $\mathrm{N}$ genes were amplified in OF from four cases, two of which were also positive by Microimmune measles IgM capture EIA. The OF and serum samples from two of these four cases were taken 1 day after onset of symptoms, and for the other two cases 2 and 3 days after onset.

Applying the criteria for a laboratory-confirmed case defined in the Methods section, 68/92 suspected cases shown in Table 1 were confirmed as measles cases. In addition to the 92 cases described in Table 1, there were seven suspected cases from whom only OF samples were available, and these were investigated by EIA and PCR. For a further four cases where matched serum and OF samples were available for investigation by EIA, three of these had sufficient OF available for PCR. Results from these 11 additional cases brought the total number of laboratory-confirmed measles cases to 75 from the 103 suspected cases investigated in this outbreak. The sensitivity, specificity, positive and negative predictive values of the Siemens' measles-specific IgM EIA on serum, OF IgM POCT, and $\mathrm{H}$ gene detection from POCT strips for the 103 suspected cases investigated are shown in Table 2. The results show that the OF IgM POCT has good sensitivity compared to the gold standard Siemens measles IgM EIA, detecting $75 \%$ of the measles cases. Furthermore, after testing OF for measles-specific IgM using the POCT, amplification of the $\mathrm{H}$ gene from the test strips detected all but two of the 72 measles cases tested.

Timing of specimen collection in relation to onset of symptoms and the method used to detect measles in cases is shown in Table 3. As indicated in the Methods section, serum samples were not available from all suspected cases for testing by EIA and sufficient OF was available for testing 99 suspected cases by POCT. Timing of specimen collection for all 103 cases is shown in the first column. For each test method, the number of positive cases detected and the total number of laboratory-confirmed cases tested is shown in the corresponding columns of Table 3 .

Of the nine suspected cases from whom specimens were taken on the day of rash onset, six were identified as measles cases by all the serological and PCR methods used indicating that detectable measles-specific 
Table 2. Performance characteristics of the Siemens measles IgM EIA, measles IgM POCT on OF and H gene PCR on nucleic acid extracted from OF tested POCT strips

\begin{tabular}{lllll}
\hline \hline Test & Sensitivity $(95 \% \mathrm{CI})$ & Specificity $(95 \% \mathrm{CI})$ & PPV $(95 \% \mathrm{CI})$ & NPV $(95 \% \mathrm{CI})$ \\
\hline $\begin{array}{l}\text { Siemen measles IgM } \\
\text { EIA on serum samples }\end{array}$ & $84 \cdot 1 \%(73 \cdot 3-91 \cdot 8)$ & $100 \cdot 0 \%(86 \cdot 8-100 \cdot 0)$ & $100 \cdot 0 \%(93 \cdot 8-100 \cdot 0)$ & $70 \cdot 3 \%(53 \cdot 0-84 \cdot 1)$ \\
OF IgM POCT & $75 \cdot 0 \%(63 \cdot 4-84 \cdot 5)$ & $96 \cdot 2 \%(80 \cdot 4-99 \cdot 9)$ & $98 \cdot 2 \%(90 \cdot 3-100 \cdot 0)$ & $58 \cdot 1 \%(42 \cdot 1-73 \cdot 0)$ \\
$\begin{array}{l}\text { H gene PCR from POCT } \\
\text { strips }\end{array}$ & $97 \cdot 2 \%(90 \cdot 3-99 \cdot 7)$ & $100 \%(87 \cdot 2-100 \cdot 0)$ & $100 \%(94 \cdot 9-100 \cdot 0)$ & $93 \cdot 1 \%(77 \cdot 2-99 \cdot 2)$ \\
\hline \hline
\end{tabular}

EIA, Enzyme immunoassay; OF, oral fluid; POCT, point of care test; CI, confidence interval; PPV, positive predictive value; NPV, negative predictive value.

Table 3. Comparison of the laboratory method used to confirm measles cases on differing days post-onset of symptoms

\begin{tabular}{|c|c|c|c|c|c|c|c|c|c|}
\hline \multirow[b]{2}{*}{$\begin{array}{l}\text { Days } \\
\text { post-onset }\end{array}$} & \multicolumn{9}{|c|}{$\begin{array}{l}\text { Detection of measles cases in relation to timing of specimen collection post onset of symptoms and method used } \\
\text { (number positive/total number laboratory-confirmed measles cases tested*) }\end{array}$} \\
\hline & $\begin{array}{l}\text { Serum } \\
\text { IgM } \\
\text { EIA }\end{array}$ & $\begin{array}{l}\text { OF } \\
\text { IgM } \\
\text { POCT }\end{array}$ & $\begin{array}{l}\text { Serum } \\
\text { IgM } \\
\text { POCT }\end{array}$ & $\begin{array}{l}\text { OF IgM } \\
\text { EIA }\end{array}$ & $\begin{array}{l}\text { H gene } \\
\text { from } \\
\text { OF }\end{array}$ & $\begin{array}{l}\text { H gene } \\
\text { POCT } \\
\text { strips }\end{array}$ & $\begin{array}{l}\mathrm{N} \text { gene } \\
\text { from } \\
\mathrm{OF}\end{array}$ & $\begin{array}{l}\text { N gene } \\
\text { POCT } \\
\text { strips }\end{array}$ & $\begin{array}{l}\text { Total laboratory- } \\
\text { confirmed cases*/total } \\
\text { suspected cases }\end{array}$ \\
\hline 0 & $6 / 6$ & $6 / 6$ & $6 / 6$ & $6 / 6$ & $6 / 6$ & $6 / 6$ & $6 / 6$ & $6 / 6$ & $6 / 9$ \\
\hline 1 & $12 / 19$ & $10 / 19$ & $11 / 19$ & $15 / 20$ & $20 / 20$ & $19 / 19$ & $20 / 20$ & $19 / 19$ & $20 / 27$ \\
\hline 2 & $6 / 8$ & $6 / 8$ & $7 / 7$ & $8 / 8$ & $7 / 8$ & $7 / 8$ & $6 / 8$ & $7 / 8$ & $8 / 10$ \\
\hline 3 & $7 / 8$ & $4 / 7$ & $7 / 8$ & $7 / 8$ & $7 / 7$ & $7 / 7$ & $7 / 7$ & $7 / 7$ & $8 / 8$ \\
\hline 4 & $9 / 9$ & $7 / 9$ & $9 / 9$ & $8 / 10$ & $10 / 10$ & $9 / 9$ & $10 / 10$ & $9 / 9$ & $10 / 11$ \\
\hline 5 & $7 / 7$ & $8 / 9$ & $6 / 6$ & $9 / 9$ & $9 / 9$ & $9 / 9$ & $9 / 9$ & $9 / 9$ & $9 / 12$ \\
\hline 6 & $2 / 2$ & $2 / 2$ & $2 / 2$ & $2 / 2$ & $2 / 2$ & $2 / 2$ & $2 / 2$ & $2 / 2$ & $2 / 2$ \\
\hline 7 & $1 / 1$ & $1 / 1$ & $1 / 1$ & $1 / 1$ & $1 / 1$ & $1 / 1$ & $1 / 1$ & $1 / 1$ & $1 / 2$ \\
\hline Unknown & $9 / 9$ & $10 / 11$ & $9 / 9$ & $10 / 10$ & $10 / 11$ & $10 / 11$ & $10 / 11$ & $11 / 11$ & $11 / 22$ \\
\hline Total & $59 / 69$ & $54 / 72$ & $58 / 67$ & $66 / 75$ & $72 / 74$ & $70 / 72$ & $71 / 74$ & $71 / 72$ & $75 / 103$ \\
\hline Sensitivity & $85 \cdot 5 \%$ & $75 \cdot 0 \%$ & $86 \cdot 6 \%$ & $88 \cdot 0 \%$ & $97 \cdot 3 \%$ & $97 \cdot 2 \%$ & $95 \cdot 9 \%$ & $98 \cdot 6 \%$ & \\
\hline
\end{tabular}

EIA, Enzyme immunoassay; OF, oral fluid; POCT, point of care test.

* Measles confirmed by $\mathrm{H}$ and/or N gene PCR directly from OF specimen or by measles specific IgM EIA on serum samples from a suspected case.

All measles cases could not be investigated in all tests because of missing or insufficient volume of specimen.

IgM antibody and viral nucleic acid were present in acute cases.

For the 27 suspected cases from whom specimens were collected 1 day post-onset of symptoms, 20 measles cases were laboratory confirmed using the criteria outlined above. However, serological methods using serum IgM EIA and serum IgM POCT each detected only $12 / 19(57 \cdot 8 \%)$ and $11 / 19 \quad(57 \cdot 8 \%)$ confirmed cases, respectively. Of the seven measles cases not detected by the serum IgM EIA, four gave repeatedly equivocal results and three were negative. The eight measles cases not detected by the serum IgM POCT included four cases giving an EIA equivocal result and two cases giving a positive EIA result. The OF IgM POCT detected measles in $10 / 19$ cases
$(52.6 \%)$ from specimens collected 1 day after onset of symptoms. Of the nine measles cases not identified by OF IgM POCT, four were equivocal, two were low positive $\left(\mathrm{OD}_{450 \mathrm{~nm}}\right.$ of 0.43 and 0.59 , test/cut-off $\left.<3.0\right)$ and two negative in the serum IgM EIA and for one case there was no serum sample available for testing.

The POCT for measles IgM on OF identified only $4 / 7$ measles cases $(57 \cdot 1 \%)$ when the specimens were collected 3 days post-onset of symptoms. One of three cases not identified by the POCT was also negative in serum IgM EIA; the other two were positive in the serum IgM EIA, and EIA on the OF from these cases indicated very high levels of measles-specific IgM, giving a test to cut-off signal $>25$. There was insufficient $\mathrm{OF}$ remaining from these two cases to 
retest these specimens after dilution to investigate if the high levels of measles-specific IgM in the specimen had sequestered the entire rNP added for the POCT reaction, leaving no epitopes available for binding to the gold-conjugated monoclonal anti-NP antibody, as was the case for one such specimen in a previous study [9].

With the exception of OF specimens collected from cases on days 1 and 3 after symptom onset, $>75 \%$ of the measles cases were identified using the OF IgM POCT at all other time points.

The OF IgM EIA detected 15/20 confirmed measles cases where specimens were taken 1 day after onset (Table 3, column 4). Two of these 15 cases were equivocal and one negative by serum IgM EIA. For specimens collected 2 days after onset of rash, the OF IgM EIA detected all eight confirmed cases, two of which were missed by the serum IgM EIA. Of the 10 confirmed measles cases where specimens were taken 4 days after rash symptoms the OF IgM EIA detected eight cases with one other case giving an equivocal result. The sensitivity of measles detection using OF IgM EIA and serum IgM EIA were similar for specimens collected at all other time points after rash presentation.

Measles $\mathrm{H}$ gene PCR performed directly from OF samples detected all but two of the 75 confirmed cases (Table 3, column 5). The two cases that were missed by PCR were serologically positive for measles IgM as outlined above. For one of these two cases OF was collected 2 days post-onset of rash and for the other case the timing was not known. All the measles cases detected by $\mathrm{H}$ gene PCR directly from OF specimens could also be detected after $\mathrm{H}$ gene PCR of nucleic acids extracted from POCT strips that had been used for detecting IgM in the OF (Table 3, column 6).

PCR on OF from the 28 non-measles suspected cases did not yield $\mathrm{H}$ and $\mathrm{N}$ gene amplicons. Measles N gene nested PCR on OF detected 72/75 measles cases. This included the two cases missed by the $\mathrm{H}$ gene PCR on $\mathrm{OF}$ and one additional case from whom the sample was taken 2 days post-onset of symptoms.

The $\mathrm{N}$ gene PCR was more sensitive when testing extracts of POCT strips previously used for OF IgM detection $(71 / 72,98 \cdot 6 \%)$ than when testing OF directly $(71 / 74,95 \cdot 9 \%)$. It detected two cases that had been PCR negative directly on OF specimens. For the first of the two cases, the date of OF collection in relation to onset of symptoms was not known. The serum IgM EIA and OF IgM EIA results on this case were positive and the $\mathrm{H}$ gene PCR result was negative. Although the $\mathrm{N}$ gene was amplified from the used POCT strips, the nucleotide sequence was not consistent with a wild-type measles strain. The $\mathrm{N}$ gene sequence obtained after amplification from the strip was identical to the measles strain used to produce the recombinant NP antigen employed in the POCT device. This case was serologically confirmed as measles, with no detectable viral RNA in the OF specimens. The second case was a 5-month-old baby from whom OF was collected 2 days post-onset of symptoms. Measles IgM was detected in both serum and OF by EIA and POCT. The measles $\mathrm{H}$ gene was not detected by realtime PCR. The $\mathrm{N}$ gene was amplified from the POCT strip, after testing OF for IgM from this case, and sequenced as wild-type measles of genotype B3.

$\mathrm{N}$ gene sequence identical to the strain used to produce the recombinant NP was also detected after N gene PCR from POCT strips of five of the 28 nonmeasles cases indicating that, in the absence of wildtype measles, trace levels of residual $\mathrm{N}$ gene present in the recombinant NP antigen amplified sporadically, as observed previously [9].

\section{Detection of measles in vaccinated individuals}

Of the 103 suspected measles cases investigated 17 had received vaccine prior to sample collection and measles was identified serologically and by PCR in two of these cases. The first of these cases was from a 9-month-old child from Mashonaland East who received measles vaccine 2 days prior to sample collection. There was a strong serological response in the measles-specific IgM EIA in serum $\left(\mathrm{OD}_{450 \mathrm{~nm}} 0.598\right.$ or a test/cut-off of $\sim 3 \cdot 0$ ) and an even stronger response in $\mathrm{OF}\left(\mathrm{OD}_{450 \mathrm{~nm}}>3 \cdot 0\right.$. test/cut-off $\left.>30 \cdot 0\right)$. Both $\mathrm{H}$ and $\mathrm{N}$ genes were amplified directly from the OF; however, the $\mathrm{N}$ gene was not sequenced and consequently it was not possible to confirm this as a wild-type measles case. The second case was a 14-year-old from Harare province who received vaccine 98 days prior to specimen collection; measles-specific IgM was equivocal in the serum sample and positive in $\mathrm{OF}$. $\mathrm{H}$ and $\mathrm{N}$ genes were amplified directly from $\mathrm{OF}$ from this case and the $\mathrm{N}$ gene sequenced and genotyped as B3 indicating a wild-type measles infection in a vaccinated individual. 


\section{Investigation of circulating rubella during the measles outbreak}

Sera from 87/96 suspected measles cases were available for testing in the rubella IgM EIA. Fifty-two of 53 measles IgM-positive cases were negative for rubella IgM and serum from the remaining case gave an equivocal result. Rubella IgM was detected in 4/ 28 measles IgM-negative cases from four different provinces in the north and east of Zimbabwe, indicating the presence of circulating rubella during this measles outbreak. The date of onset of rash symptoms was not known for all four rubella cases. The ages of three rubella cases were known: all were aged between 5 and 6 years, and all three had received measles containing vaccine $>129$ days prior to serum sample collection. The four rubella IgM-positive cases and an additional four cases that gave rubella IgM equivocal results were negative by POCT and by PCR for measles $\mathrm{H}$ and $\mathrm{N}$ genes.

\section{Genotyping}

Amplicons for sequence analysis were selected to ensure that all geographical provinces from which PCR-positive specimens had been collected were represented. This included 10 cases from Harare, nine from Mashonaland East, five from Midlands, four from Chitungwiza and one each from Mashonaland West and Masvingo. For each of the 24 cases sequenced, an identical measles strain was identified in the matched OF and POCT strip. All 30 cases were identified as genotype B3. Representative sequences were submitted to GenBank (KP232929-KP232958).

\section{DISCUSSION}

Large outbreaks of measles are still occurring in many parts of the world, despite the availability of an inexpensive and effective vaccine. In recent ProMed mail measles update reports for 2014 [17, 18], between January and June 2014, 5445 and 4000 suspected measles cases were reported in the Philippines and Somalia, respectively, and in the first week of August 2014, 160 suspected measles cases were reported nationwide in Ethiopia. Most of these cases were in young unvaccinated children. Serological confirmation of suspected measles cases enables effective control measures to be implemented. Zimbabwe experienced a nationwide outbreak of measles in
September 2009 which continued until November/ December 2010, and a nationwide vaccination campaign to contain the disease was conducted between late May 2010 and early June 2010. Both blood and OF samples were collected to confirm suspected cases. The processed samples were tested by commercially available EIA to confirm measles cases. This afforded the opportunity to evaluate our POCT for measles in epidemiological circumstances in which we would anticipate it would be deployed.

Serological tests for measles that can be performed at the point of contact, in field settings, are likely to improve surveillance and the speed of response to any outbreaks. Serum samples are not ideal for fieldbased applications since they involve the collection of blood by venepuncture, which is often resisted by parents and young children, difficult to obtain from very young children and requires well-trained staff and laboratory facilities to process. Surveillance of measles would be improved if the method of sample collection was simplified. Ideally, serological tests that use non-invasive specimens such as OF, rather than blood, would be preferable. In this study we confirmed previous findings that $\mathrm{OF}$ can substitute for serum samples for EIA detection of measlesspecific IgM without significantly reducing sensitivity or specificity [19]. The ease with which OF can be collected painlessly by relatively untrained personnel, the high compliance rates among subjects being investigated and the relatively lower risk of HIV and hepatitis transmission to healthcare workers compared to venepuncture equipment makes OF the ideal specimen for serological investigations in measles control programmes. Furthermore, measles-specific nucleic acids can be amplified by PCR from OF and sequenced to enable genotyping of outbreak strains and tracking of the modes of transmission.

From the day of onset to day 7 sensitivity of measles-specific IgM detection was similar for both the OF POCT and serum EIA. The level of measlesspecific IgM produced in acute cases appears to be sufficient for sensitive detection by the OF POCT. In cases where the IgM levels are low in serum, such as in cases where the serum EIA gives equivocal results, specific antibodies were not detected in OF by the OF POCT, but were often detected by the OF EIA illustrating the relative difference in sensitivity of these two methods. Exceptionally high concentrations of measles-specific IgM antibody in OF can occasionally give rise to OF POCT false-negative results, as was found in this study for two cases from whom the $\mathrm{OF}$ 
sample was collected 3 days post-onset of symptoms. This phenomenon has also been observed in a previous study [9] and is probably the result of the current configuration of the POCT, whereby OF specimen and rMVN antigen is pre-mixed before application to the POCT strips. Reconfiguring this test by directly attaching the rMVN antigen to the gold particles should overcome the problem of antigen sequestration observed with the current method and improve further the sensitivity of the OF POCT.

The lower sensitivity of the POCT on OF compared to serum EIA is not an epidemiologically significant limitation for measles control programmes. The sensitivity is likely to be sufficient for case-based surveillance, although some true cases will not be confirmed as measles. However, even with a slightly lower sensitivity than serum EIA, measles outbreaks will still be detected by the OF POCT because the WHO algorithm recommends that a minimum of 5-10 samples be tested from a suspected outbreak to confirm the cause as measles [20]. For example, in this study in Chitungwiza samples were collected from seven suspected cases on 23 June 2010, two of these were negative by $\mathrm{H}$ gene $\mathrm{PCR}$ on $\mathrm{OF}$, as were the matched serum by Siemens IgM EIA. On the other hand, of the five measles cases confirmed by PCR and EIA, three were positive using the POCT on $\mathrm{OF}$, illustrating that in practice measles outbreaks could be confirmed at the point of contact using such a POCT device on a non-invasive specimen, i.e. OF.

We confirmed our previous findings that after testing OF for measles-specific IgM using POCT, it was possible to amplify measles genes from test strips and identify the infecting measles genotype and strain. There was no loss in sensitivity of detection of the measles $\mathrm{H}$ gene from POCT strips compared to detection directly from OF samples. This, together with the stability of viral RNA on dried POCT strips $[9,13]$, suggests that using POCT provides a rapid method for outbreak investigation in field settings, without compromising the overall sensitivity of measles confirmation because the POCT strips can subsequently be shipped to be investigated by PCR in a laboratory setting.

In conclusion, the sensitivity and specificity of the POCT for measles has the potential to be used in field settings to identify measles outbreaks since we have demonstrated that acute cases of measles can be detected on the day of presentation of symptoms in an acceptable proportion of cases. In the current configuration, our POCT is not yet field ready.
Further development of the POCT will be required to eliminate the pre-mixing of $\mathrm{OF}$ samples with measles antigen. This, together with development of an OF collection device that can deliver the sample directly to the POCT device, would make the test an invaluable tool for the control and surveillance of measles. These further developments are currently being investigated and preliminary results are encouraging.

\section{ACKNOWLEDGEMENTS}

The authors thank Professor John Parry for his critical review of the manuscript. This work received no specific grant from any funding agency, commercial or not-for-profit sectors. WHO/HQ provided funding for travel and subsistence for A.S.'s visit to the UK laboratory.

\section{DECLARATION OF INTEREST}

D. Samuel is a director of Microimmune Limited. Measles specific IgM tests on OF from suspected cases in the Zimbabwe outbreak were investigated using Microimmune EIA test kits. R. Slibinskas is a director of UAB Baltymas. UAB Baltymas is a biotechnology company specialising in production of recombinant proteins for diagnostic and therapeutic use.

\section{REFERENCES}

1. WHO. Global measles and rubella strategic plan: 2012-2020 (http://www.who.int/immunization/news room/Measles_Rubella_StrategicPlan_2012_2020.pdf).

2. Erdman D, et al. Evaluation of monoclonal antibodybased capture enzyme immunoassays for detection of specific antibodies to measles virus. Journal of Clinical Microbiology 1991; 29: 1466-1471.

3. Ratnam S. et al. Performance of indirect immunoglobulin (IgM) serology tests and IgM capture assays for the laboratory diagnosis of measles. Journal of Clinical Microbiology 2000; 38: 99-104.

4. Uzicanin A. et al. Dried blood spots on filter paper as an alternative specimen for measles diagnostics: detection of measles immunoglobulin $\mathrm{M}$ antibody by a commercial enzyme immunoassay. Journal of Infectious Diseases 2001; 204, S564-S569.

5. Riddell M, et al. Detection of measles virus-specific immunoglobulin $\mathrm{M}$ in dried venous blood samples by using a commercial enzyme immunoassay. Journal of Clinical Microbiology 2002; 40: 5-9

6. Perry K, et al. Detection of measles, mumps and rubella antibodies in saliva using antibody capture radioimmunoassay. Journal of Medical Virology 1993; 40: 235-240 
7. Jin L et al. The diversity of measles virus in the United Kingdom, 1992-1995. Journal of General Virology 1997; 78: $1287-1294$

8. Nigatu W et al. Measles virus strains circulating in Ethiopia 1998-1999; molecular characterisation using oral fluid samples and identification of a new genotype. Journal of Medical Virology 2001; 65: 373-380.

9. Warrener $\mathbf{L}$, et al. A point-of-care test for measles diagnosis: detection of measles-specific IgM antibodies and viral nucleic acid. Bulletin of the World Health Organisation 2011; 89: 675-682.

10. WHO. Manual for the Laboratory Diagnosis of Measles and Rubella Virus Infection, 2nd edn. WHO/IVB/07.01. Geneva, Switzerland: World Health Organization, 2007.

11. Boom R, et al. Rapid and simple method for purification of nucleic acids. Journal of Clinical Microbiology 1990; 28: 495-503.

12. Stockton J, et al. Multiplex PCR for typing and subtyping influenza and respiratory syncytial viruses. Journal of Clinical Microbiology 1998; 36: 2990-2995.

13. Warrener L, et al. Development and evaluation of a rapid immunochromatographic test for mumps-specific IgM in oral fluid specimens and use as a matrix for preserving viral nucleic acid for RT-PCR. Journal of Medical Virology 2010; 82: 485-493.
14. Thomas B, et al. Development and evaluation of a realtime PCR assay for rapid identification and semiquantitation of measles virus. Journal of Medical Virology 2007; 79: 1587-1592.

15. Kühne M, Brown DW, Jin L. Genetic variability of measles virus in acute and persistent infections. Infection, Genetics and Evolution 2006; 6: 269-276.

16. WHO. New genotype of measles virus and update on global distribution of measles genotypes (update). Part 1. Weekly Epidemiological Record 2005; 80: 347-351.

17 ProMed mail. Measles update (32). ProMed-mail 10 August 2014: 20140810.2676781 (http://www.promed mail.org). Accessed 10 August 2014.

18. ProMed mail. Measles update (33). ProMed-mail 17 August 2014: 20140817.2698791 (http://www.promedmail.org). Accessed 17 August 2014.

19. Samuel D, et al. Development of a measles specific IgM ELISA for use with serum and oral fluid samples using recombinant measles nucleoprotein produced in Saccharomyces cerevisiae. Journal of Clinical Virology 2003; 28: 121-129.

20. WHO. Mortality reduction and regional elimination: strategic plan 2001-2005; global measles. Geneva: World Health Organisation, 2001, pp. 1-31. 